Artículo científico

Volumen 32(3):901-919. Septiembre-diciembre, 2021 e-ISSN 2215-3608, doi:10.15517/am.v32i3.43313 https://revistas.ucr.ac.cr/index.php/agromeso/index

\title{
Sistemas silvopastoriles: mitigación de gases de efecto invernadero, bosque seco tropical - Colombia ${ }^{1}$
}

\section{Silvopastoral systems: Mitigation of greenhouse gases in the Tropical Dry Forest - Colombia}

\author{
José Luis Contreras-Santos ${ }^{2}$, Judith Martinez-Atencia ${ }^{2}$,Bindu Raghavan ${ }^{3}$, Leonardo Lopez-Rebolledo ${ }^{2}$, \\ Jeyson Garrido-Pineda
}

1 Recepción: 3 de agosto, 2020. Aceptación: 4 de marzo, 2021. Este trabajo fue parte del proyecto "Estrategias para mejorar la competitividad y sostenibilidad de producción de carne y leche en el Caribe húmedo", financiado por el Ministerio de Agricultura y Desarrollo Rural a través del Convenio No. 1828, y se llevó a cabo en la Corporación Colombiana de Investigación Agropecuaria (AGROSAVIA), en el Centro Investigación Turipaná, Montería, Córdoba, Colombia.

2 Corporación Colombiana de Investigación Agropecuaria (AGROSAVIA), Centro de Investigación Turipaná, Km 13 Vía Montería-Cereté, Colombia.jlcontreras@agrosavia.co (autor para la correspondencia, https://orcid.org/0000-0002-8179-3430); jcmartinez@agrosavia.co (https://orcid.org/0000-0002-8275-2956); lalopezr@agrosavia.co (https://orcid.org/0000-0002-9099-1957); jfgarrido@agrosavia.co (https:// orcid.org/0000-0002-1405-1066).

3 Consultora independiente PhD, New Delhi, India. bindugnape@gmail.com (https://orcid.org/0000-0002-1819-5251).

\section{Resumen}

Introducción. Los sistemas silvopastoriles (SSP) juegan un papel destacado en la captura de carbono en el suelo y mitigación de gases de efecto invernadero. Objetivo. Cuantificar los servicios ecosistémicos ofrecidos por cuatro SSP en comparación con una pradera con gramíneas, en el valle medio del río Sinú, en Colombia. Materiales y métodos. Se midieron los flujos de metano y óxido nitroso mediante el uso de cámaras cerradas, durante un periodo de ocho semanas consecutivas (septiembre a noviembre, 2013). También se midió el carbono orgánico en el suelo a dos profundidades (0-5 y 5-15 cm), por el método de combustión y se determinaron variables físicoquímicas del suelo. El diseño correspondió a bloques completos al azar con cinco tratamientos y tres repeticiones. Los tratamientos correspondieron a cuatro sistemas silvopastoriles conformados por diferentes componentes arbóreos: Tectona grandis (SSP1), Tabebuia rosea (SSP2), Pachira quinata (SSP3) y Acacia mangium (SSP4), en comparación a una pradera con solo gramínea (Megathyrsus maximus cv. Mombasa). Resultados. Los mayores contenidos de materia orgánica, fósforo y calcio se

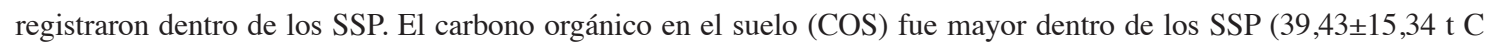
$\left.\mathrm{ha}^{-1}\right)$ en comparación con la pradera $\left(33,43 \pm 17,63 \mathrm{t} \mathrm{C} \mathrm{ha}^{-1}\right)$. Los SSP se comportaron la mayor parte del tiempo como sumideros de metano, al inmovilizar en promedio $-460 \pm 0,42 \mu \mathrm{g} \mathrm{CH}_{4} \mathrm{~m}^{-2} \mathrm{~h}^{-1}$. Las menores tasas de emisión de óxido nitroso se evidenciaron dentro de los sistemas SSP1 y SSP2 (460 $\pm 0,60$ y $620 \pm 1,19 \mu \mathrm{g} \mathrm{N}_{2} \mathrm{O} \mathrm{m}^{-2} \mathrm{~h}^{-1}$, respectivamente). Conclusión. La implementación de SSP contribuye en la disminución de procesos degradativos (físicos y químicos) del suelo, al aumento de las reservas de carbono del suelo y, por consiguiente, son una estrategia de mitigación de gases de efecto invernadero en los sistemas ganaderos.

Palabras claves: gases de efecto invernadero, mitigación, propiedades del suelo, carbono orgánico en el suelo, producción ganadera. 


\begin{abstract}
Introduction. Silvopastoral systems (SSP) play a leading role in soil carbon sequestration and greenhouse gas mitigation. Objective. To quantify the ecosystem services provided by four SSPs in comparison with a grass pasture in the middle valley of the Sinú river in Colombia. Materials and methods. Methane and nitrous oxide fluxes were measured using closed chambers, during a period of eight consecutive weeks (September to November, 2013). Soil organic carbon was also measured at two depths (0-5 and 5-15 cm), by the combustion method, and soil physicochemical variables were determined. The design corresponded to randomized complete blocks with five treatments and three replications. The treatments corresponded to four silvopastoral systems made up of different tree components: Tectona grandis (SSP1), Tabebuia rosea (SSP2), Pachira quinata (SSP3) and Acacia mangium (SSP4), compared to a grass-only meadow (Megathyrsus maximus cv Mombasa). Results. The highest contents of organic matter, phosphorus, and calcium were registered within the SSP. Soil organic carbon (COS) was higher within the SSPs $\left(39.43 \pm 15.34 \mathrm{t} \mathrm{C} \mathrm{ha}^{-1}\right)$, compared to the grassland $\left(33.43 \pm 17.63 \mathrm{t} \mathrm{C} \mathrm{ha}^{-1}\right)$. The SSPs behaved most of the time as methane sinks, immobilizing on average $-460 \pm 0.42 \mu \mathrm{g} \mathrm{CH}_{4} \mathrm{~m}^{-2} \mathrm{~h}^{-1}$. The lowest nitrous oxide emission rates were evident within SSP1 and SSP2 systems (460 $\pm 0.60 ; 620 \pm 1.19 \mu \mathrm{g} \mathrm{N}_{2} \mathrm{O} \mathrm{m}^{-2} \mathrm{~h}^{-1}$, respectively). Conclusion. The implementation of SSP contributes to the decrease of soil degradative processes (physical and chemical), to the increase of soil carbon stocks and, consequently, they are a greenhouse gas mitigation strategy in livestock systems.
\end{abstract}

Keywords: greenhouse gases, mitigation, soil properties, soil organic carbon, livestock production.

\title{
Introducción
}

A nivel mundial las emisiones antropogénicas totales de gases de efecto invernadero (GEI) aumentaron entre 1970 y 2010, con mayor incremento absoluto entre los años 2000 y 2010 , con aumento de $+2,2 \%$ año $^{-1}$, lo cual ha sido atribuido en parte al crecimiento demográfico y económico (Pachauri et al., 2014). A pesar del creciente número de políticas de mitigación del cambio climático establecidas por los diferentes gobiernos, las emisiones relacionadas a estos procesos alcanzaron cifras alarmantes en el año $2010\left(49 \pm 4,5 \mathrm{Gt} \mathrm{CO}_{2} \text {-eq año-1 }\right)^{-1}$ Dichas emisiones se han detectado en todo el sistema climático y es probable que hayan sido la causa del calentamiento observado a partir de la segunda mitad del siglo XX (Pachauri et al., 2014).

Los sistemas agropecuarios contribuyen de forma significativa con la emisión de tres de los principales gases de efecto invernadero (GEI): metano $\left(\mathrm{CH}_{4}\right)$, dióxido de carbono $\left(\mathrm{CO}_{2}\right)$ y óxido nitroso $\left(\mathrm{N}_{2} \mathrm{O}\right)$ (Hernández \& Corona, 2018; Pachauri et al., 2014). Algunos reportes atribuyen a la ganadería el $53 \%$ de las emisiones de metano $\left(\mathrm{CH}_{4}\right)$ del sector agropecuario, proveniente en su mayoría de la fermentación entérica de los rumiantes (Charmley et al., 2016). Otros reportes indican que el $29 \%$ de las emisiones de óxido nitroso $\left(\mathrm{N}_{2} \mathrm{O}\right)$, están relacionadas con procesos de fertilización ureica que ocurren en la agricultura (Gerber et al., 2013). De igual forma, el cambio en los patrones de uso del suelo, a causa de la transformación de áreas boscosas, en suelos con pastizales, ha conllevado a la reducción del carbono acumulado en el suelo, lo que genera emisiones de GEI hacia la atmósfera (Pérez-Ramírez et al., 2013).

La tendencia a nivel mundial ha sido la búsqueda de alternativas para mitigar los procesos degradativos del suelo y reducir las emisiones de GEI, para lo cual, una de las estrategias propuestas ha sido la implementación de los sistemas agroforestales y silvopastoriles (Montagnini et al., 2015; Murgueitio-Restrepo et al., 2016). En el caso de la ganadería en el trópico, el enfoque de la estrategia ha sido el secuestro del carbono y el aumento de la biodiversidad, por lo que los sistemas silvopastoriles (SSP) han sido vistos como una estrategia fundamental para 
una producción más sostenible, en especial frente a las perspectivas del cambio climático (Alonso, 2011). Los SSP son considerados herramientas claves en la transformación de la agricultura convencional hacia una agricultura climáticamente inteligente, que aumente la productividad en forma sostenible y resiliente (capacidad de adaptación ante los diferentes impactos ambientales o antropogénicos), que a la vez reduzca, evite o disminuya los GEI (Jose \& Dollinger, 2019; Organización de las Naciones Unidas para la Alimentación y la Agricultura \& Grupo Técnico intergubernamental de Suelos, 2015).

La implementación de SSP en la ganadería tropical, implica un cambio fundamental en el uso del suelo, al pasar de los sistemas ganaderos tradicionales, que se basan en una dieta animal conformada por solo gramíneas, a sistemas que asocien las especies gramíneas con componentes arbóreos, con lo cual se aumenta la acumulación de carbono orgánico en el suelo (COS), debido al volumen de material orgánico que aportan las especies (ContrerasSantos et al., 2019; De-Stefano \& Jacobson, 2017; Dollinger \& Jose, 2019).

El presente estudio se llevó a cabo con el objeto de cuantificar los servicios ecosistémicos ofrecidos por cuatro sistemas silvopastoriles (SSP), en comparación con una pradera con gramíneas, en el Valle medio del Río Sinú, en Colombia.

\section{Materiales y métodos}

\section{Locación}

La investigación se realizó durante un periodo de ocho semanas, comprendidas entre septiembre y noviembre de 2013, bajo las condiciones del centro de investigación Turipaná de la Corporación Colombiana de Investigación Agropecuaria (AGROSAVIA), localizado en el municipio de Cereté, en el departamento de Córdoba, Colombia, con coordenadas geográficas $75^{\circ} 47^{\prime} \mathrm{W}, 8^{\circ} 50^{\prime} \mathrm{N}$. El centro de investigaciones se encuentra a $15 \mathrm{msnm}$, temperatura promedio anual de $28{ }^{\circ} \mathrm{C}$, precipitación promedio anual de $1200 \mathrm{~mm}$ y humedad relativa del $82 \%$. Las precipitaciones en la zona presentan distribución bimodal, con dos periodo lluvioso (abril a noviembre) y seco (diciembre a marzo). De acuerdo con lo anterior, la investigación se realizó durante el periodo lluvioso del año, según la clasificación de zonas de vida realizadas por Holdridge (2000), el área de estudio pertenece al bosque seco tropical (Bs T).

El suelo del área experimental corresponde a un Vertic Endoaquepts (Soil Survey Staff, 2014), perteneciente a la serie La Pozona, el cual presenta predomino de textura arcillosa, de tipo expansiva (2:1), régimen de humedad údico y temperatura isohipertérmica, con secuencia de horizontes Ap, Bw, Bg y Bg2 (Figura 1).

\section{Tratamientos}

Los tratamientos silvopastoriles evaluados y la pradera con solo gramínea se describen en Cuadro 1.

El área experimental utilizada para esta investigación, había sido establecida en el Centro de Investigaciones Turipaná desde el año 2006, con una distancia de siembra de 3,5 m entre surcos y 3,5 m entre plantas, para una densidad de población de 816 árboles ha ${ }^{-1}$. Seis años después del establecimiento (2012) de estos forestales, se realizó una entresaca con una presión del $30 \%$ de la densidad inicial. Posterior a la entresaca, se estableció la pastura (M. maximus cv. Mombasa) entre las líneas forestales. El área experimental se dividió en quince potreros de $310 \mathrm{~m}^{2}$, con el fin de establecer un ciclo de pastoreo rotacional de 22,5 días (1,5 días de ocupación y 21 días de descanso). Los potreros se ocuparon con una carga de tres animales por hectárea (3 UGG ha-1), con el genotipo Brahman o Cebú comercial, con peso promedio inicial de $200 \pm 15 \mathrm{~kg} \mathrm{animal}^{-1}$. El área experimental total fue de $4650 \mathrm{~m}^{2}$, con una disponibilidad de forraje de las gramíneas de alrededor de 1200 a $1300 \mathrm{~kg} \mathrm{MS} \mathrm{ha}^{-1} \mathrm{año}^{-1}$. 


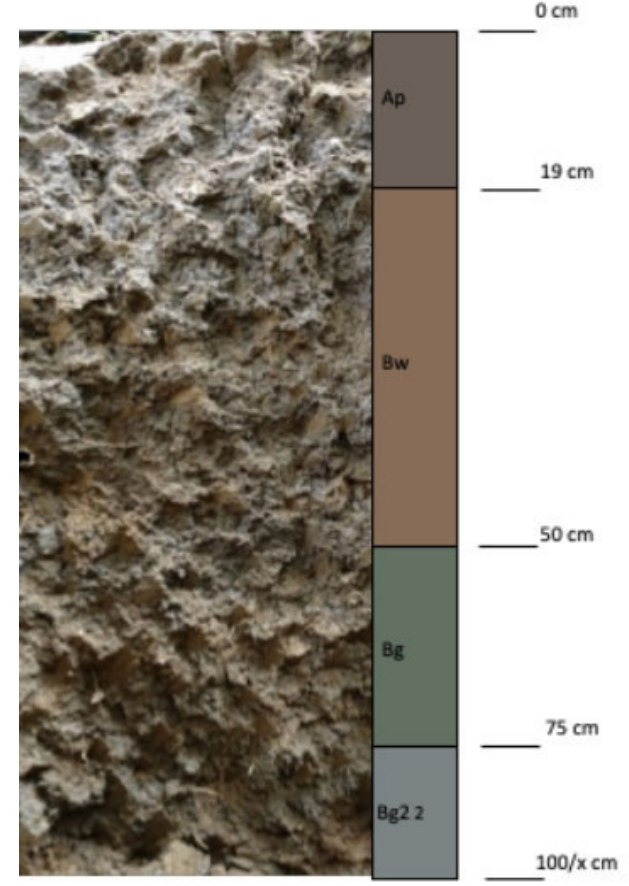

Figura 1. Perfil modal del suelo bajo los sistemas silvopastoriles evaluados. Centro de Investigación Turipaná, Corporación Colombiana de Investigación Agropecuaria (AGROSAVIA). 2013. Municipio de Cereté, departamento de Córdoba, Colombia.

Figure 1. Modal profile of the soil under the silvopastoral systems evaluated. Turipaná Research Center, Corporacion Colombiana de Investigacion Agropecuaria (AGROSAVIA). 2013. Cerete municipality, Cordoba department, Colombia.

Cuadro 1. Arreglos silvopastoriles evaluados. Centro de Investigación Turipaná, Corporación Colombiana de Investigación Agropecuaria (AGROSAVIA). Municipio de Cereté, departamento de Córdoba, Colombia. 2013.

Table 1. Silvopastoral arrangements evaluated. Turipaná Research Center, Corporacion Colombiana de Investigacion (AGROSAVIA). Cerete municipality, Cordoba department, Colombia. 2013.

\begin{tabular}{ll}
\hline Arreglo & Especies \\
\hline Pr & Gramínea Megathyrsus maximus cv. Mombasa \\
SSP1 & Tectona grandis + Megathyrsus maximus cv. Mombasa \\
SSP2 & Tabebuia rosea + Megathyrsus maximus cv. Mombasa \\
SSP3 & Pachira quinata + Megathyrsus maximus cv. Mombasa \\
SSP4 & Acacia mangium + Megathyrsus maximus cv. Mombasa \\
\hline
\end{tabular}

\section{Diseño experimental}

Se utilizó un diseño de bloques completos al azar (BCA) con cinco tratamientos y tres repeticiones. El criterio de bloqueo correspondió a la condición (bien, moderado e imperfecto y mal drenado) y dirección del drenaje del suelo. Cada unidad experimental estuvo conformada por un área de $310 \mathrm{~m}^{2}$, para un total de $4650 \mathrm{~m}^{2}$ en todo el experimento. 


\section{Variables evaluadas}

\section{Ambientales}

Durante la época de evaluación se realizó un monitoreo diario de las variables climáticas, mediante una estación meteorológica instalada en las proximidades del lote experimental. De acuerdo con los datos de precipitación obtenidos, se estableció que durante el periodo de evaluación (septiembre a noviembre del año 2013), se presentó la transición de la temporada lluviosa a la temporada seca, con disminución paulatina de la cantidad de lluvias (Figura 2).

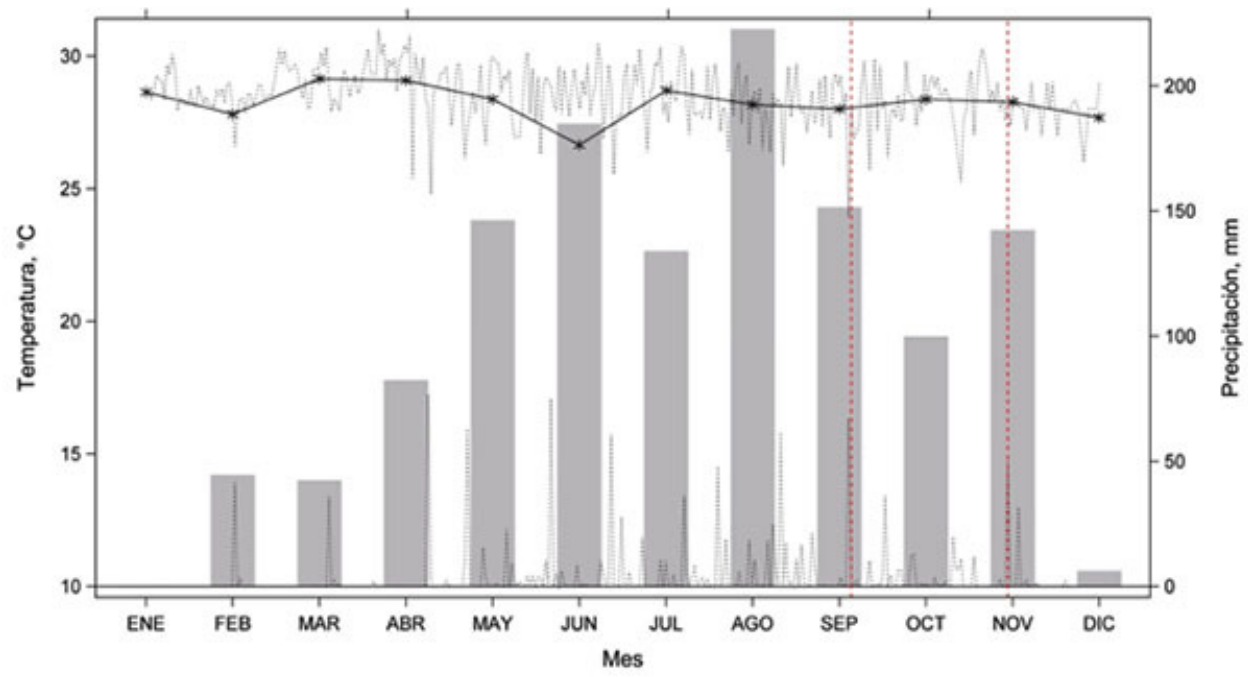

Figura 2. Parámetros climáticos (precipitación - mm y temperatura $-{ }^{\circ} \mathrm{C}$ ) del período de estudio, estación climática del Centro de Investigación Turipaná - Corporación Colombiana de Investigación Agropecuaria (AGROSAVIA), Municipio de Cereté, departamento de Córdoba, Colombia. 2013.

Las líneas punteadas de color rojo verticales representan el periodo de evaluación de los flujos de gases de efecto invernadero.

Figure 2. Climatic parameters (precipitation - $\mathrm{mm}$ and temperature - ${ }^{\circ} \mathrm{C}$ ) of the study period, climatic station of the Turipana Research Center - Corporación Colombiana de Investigación Agropecuaria (AGROSAVIA). Cerete municipality, Cordoba department, Colombia. 2013.

The vertical red dotted lines represent the evaluation period of greenhouse gas flows.

\section{Propiedades del suelo}

Se determinaron las propiedades fisicoquímicas del suelo, mediante muestras tomadas por triplicado un año después al establecimiento de la gramínea, cada $5 \mathrm{~cm}$, hasta una profundidad de $25 \mathrm{~cm}$, sobre la zona central de cada unidad experimental. También se determinó la resistencia mecánica del suelo a la penetración cada 2,5 cm hasta una profundidad de $80 \mathrm{~cm}$, con un penetrógrafo Stiboka (Eijkelkamp Soil \& Water), las lecturas se hicieron por triplicado en cada unidad experimental. La densidad aparente del suelo (Da) se determinó con el método del 
cilindro de volumen conocido (soil core), en el cual la muestra obtenida se secó en un horno a $105{ }^{\circ} \mathrm{C}$ durante $24 \mathrm{~h}$ y luego se pesó. La densidad aparente (Da) se obtuvo mediante la siguiente relación (Ecuación 1).

$$
D a=\frac{M s s}{V c} \quad \text { Ecuación } 1 .
$$

Donde, Da: densidad aparente $\left(\mathrm{g} \mathrm{cm}^{-3}\right)$; Mss: masa de suelo seco a $105{ }^{\circ} \mathrm{C}(\mathrm{g})$; Vc: volumen del cilindro $\left(\mathrm{cm}^{3}\right)$.

La humedad del suelo se determinó con periodicidad semanal, durante la evaluación de los flujos de GEI, por el método gravimétrico, la densidad real por el método del Picnómetro, la porosidad total calculada de forma indirecta mediante la relación entre la densidad aparente y la densidad real, según la metodología descrita por el Instituto Geográfico Agustín Codazzi (IGAC) (2006) (Ecuación 2).

$$
P t=\left(1-\frac{D a}{D r}\right) \times 100 \quad \text { Ecuación } 2 .
$$

Donde, Pt: porosidad total (\%), Da: densidad aparente $\left(\mathrm{g} \mathrm{cm}^{-3}\right)$ y Dr: densidad real $\left(\mathrm{g} \mathrm{cm}^{-3}\right)$. El porcentaje de la porosidad ocupada por agua (water filled porosity space) se determinó siguiendo la metodología propuesta por Espinosa-Carvajal et al. (2020) (Ecucación 3):

$$
W F P S=\left(\frac{H u m \times D a}{P t}\right) \times 100 \quad \text { Ecuación } 3 .
$$

Donde, WFPS: porcentaje de la porosidad ocupada por agua (\%); Hum: humedad gravimétrica $\left(\mathrm{g} \mathrm{g}^{-1}\right)$, Da: densidad aparente $\left(\mathrm{g} \mathrm{cm}^{-3}\right)$ y Pt: porosidad total $\left(\mathrm{g} \mathrm{g}^{-1}\right)$.

Las propiedades químicas del sitio experimental, se determinaron mediante muestras por triplicado tomadas a una profundidad de 0 a $20 \mathrm{~cm}$, las cuales se homogenizaron obteniendo $1 \mathrm{~kg}$ de suelo por unidad experimental. Estas se analizaron en el laboratorio de suelos de AGROSAVIA, con base en las metodologías propuestas por el ICAG (2006). La reacción del suelo o pH, se determinó mediante el método potenciométrico, con relación 1:1 P/V (Hendershot et al., 2007), Norma Técnica Colombiana (NTC) 5264, materia orgánica (MO) (\%) por oxidación Walkley - Black (Nelson \& Sommers, 1983), fósforo (P) $\left(\mathrm{mg} \mathrm{kg}^{-1}\right)$ por extracción Bray II modificado y cuantificación por reducción con ácido ascórbico (Bray \& Kurtz, 1945), Azufre (S) ( $\mathrm{mg} \mathrm{kg}^{-1}$ ), por extracción con monofosfato de calcio $0,008 \mathrm{M}$ y cuantificación turbidimétrica, potasio $(\mathrm{K})$, calcio $(\mathrm{Ca})$, magnesio $(\mathrm{Mg})$ y sodio (Na) $\left(\mathrm{cmol}_{(+)} \mathrm{kg}^{-1}\right)$, por medio de espectrofotometría de absorción y emisión atómica (Chapman, 2016; Hendershot et al., 2007), NTC 5349, hierro (Fe), manganeso (Mn), zinc ( $\mathrm{Zn})$ y cobre $(\mathrm{Cu})\left(\mathrm{mg} \mathrm{kg}^{-1}\right)$, se determinaron por medio del método de Olsen modificado, espectrofotometría y cuantificación por absorción atómica (NTC 5526:2007) y Boro (B) $\left(\mathrm{mg} \mathrm{kg}^{-1}\right)$ por extracción con fosfato monobásico de calcio - Azometina H (NTC 5404).

\section{Carbono orgánico acumulado en el suelo (COS)}

Para la determinación del COS se utilizó el método planteado por la Organización de las Naciones Unidas para la Alimentación y la Agricultura (Food and Agriculture Organization, 2017) (ecuación 4).

$$
\text { COS }=C O \times \text { Da } \times \text { Prof } \times \text { CFpedr } \quad \text { Ecuación } 4 .
$$

Donde, COS: stock de carbono orgánico en el suelo ( $\left(\mathrm{ha}^{-1}\right)$; C: contenido total de carbono orgánico del suelo, determinado en laboratorio por el método de oxidación Walkley - Black; Da: densidad aparente $\left(\mathrm{g} \mathrm{cm}^{-3}\right)$; Prof.: 
profundidad de muestreo o intervención y CFpedr.: coeficiente de corrección por pedregosidad (1 - \%pedregosidad) 100). Para la obtención de este último parámetro se tomaron muestras a dos profundidades de referencia 5 y $15 \mathrm{~cm}$ y se obtuvo la pedregosidad por conteo visual. Para el caso del sitio experimental, el porcentaje de pedregosidad fue igual a cero, por lo que el CFpedr fue igual a 1.

\section{Flujo de gases efecto invernadero (GEI)}

El flujo de metano $\left(\mathrm{CH}_{4}\right)$ y óxido nitroso $\left(\mathrm{N}_{2} \mathrm{O}\right)$, se monitoreó cada ocho días, por un periodo de tres meses (noventa días), comprendido entre los meses de septiembre y noviembre de 2013. Para esto, se utilizó la técnica de cámara cerrada descrita por Rondón (2000), validada y estandarizada en el Centro Internacional de Agricultura Tropical (CIAT) por Chu et al. (2007) y utilizada por Pastrana et al. (2011). Dentro de cada unidad experimental se instalaron tres cámaras cerradas. Las cámaras cerradas estaban compuestas por dos cilindros de PVC de $25 \mathrm{~cm}$ de diámetro. El primer cilindro con una altura de $7,5 \mathrm{~cm}$, se insertó en el suelo al inicio del experimento, a una profundidad de $5,5 \mathrm{~cm}$. El segundo cilindro, de $10 \mathrm{~cm}$ de altura, se instaló sobre el primer cilindro al momento de realizar la evaluación y se unió al primero mediante bandas de caucho, que generó un ambiente hermético en el interior de la cámara. El segundo cilindro tenía una tapa, con dos agujeros cubiertos con tapones de goma, uno de los cuales se usó para tomar muestras de metano y óxido nitroso y el segundo, para equilibrar la presión ambiental y registrar la temperatura del aire dentro de la cámara, al momento de la medición. En cada unidad experimental se instalaron al azar tres cámaras para monitorear cada semana el flujo de metano y óxido nitroso.

Para la determinación de estos gases, se tomaron muestras del aire dentro de la cámara con la ayuda de jeringas prácticas de $20 \mathrm{ml}$, a los intervalos de tiempo 0, 15, 30 y 45 minutos después de la instalación del cilindro superior. El contenido de la jeringa se depositó en viales cerrados en forma hermética de $15 \mathrm{ml}$, liofilizados y envasados al vacío (Rondón, 2000). Las muestras obtenidas se llevaron al laboratorio de servicios ambientales del CIAT (Palmira, Colombia), en donde se determinaron las concentraciones de los gases en partes por millón en cada una de las muestras, mediante la utilización de un cromatógrafo de gases. Con los datos obtenidos se calculó el flujo de cada gas en función del área y el tiempo, con la utilización de la siguiente relación (Ecuación 5):

$$
F=\frac{d x}{d t} \times \frac{V \times M}{A \times V m} \quad \text { Ecuación } 5 .
$$

Donde, F: flujo del gas evaluado $\left(\mathrm{CH}_{4}\right.$ y $\left.\mathrm{N}_{2} \mathrm{O}, \mu \mathrm{g} \mathrm{CH}_{4} \mathrm{~m}^{-2} \mathrm{hr}^{-1}\right)$ por unidad de área y tiempo; dx/dt: variación del gas $\left(\mathrm{CH}_{4}\right.$ y $\left.\mathrm{N}_{2} \mathrm{O}\right)$ en el aire dentro de la cámara, expresado en $\mathrm{ppm} / \mathrm{min}$; V: volumen del gas expresado en litros; A: área de la cámara en $\mathrm{m}^{2}$; $\mathrm{M}$ : masa molar de $\mathrm{N}$ por cada $\mathrm{Mol}$ de $\mathrm{N}_{2} \mathrm{O}$ o masa molar de $\mathrm{C}$ por cada mol de $\mathrm{CH}_{4}$; $\mathrm{Vm}$ : volumen molecular del gas. El volumen molar $(\mathrm{Vm})$ del gas se obtuvo mediante la siguiente relación (Ecuación 6):

$$
V m=n \times R \times{ }^{T} / P \quad \text { Ecuación } 6 .
$$

Donde, $\mathrm{n}=$ moles, $(1 \mathrm{~mol}$ del gas $)$; $\mathrm{R}$ : constante de gases ideales $\left(0,0820574587 \mathrm{~L} \mathrm{~atm} \mathrm{~K}^{-1} \mathrm{~mol}^{-1}\right)$; T: temperatura dentro de la cámara al momento de la medición $\left({ }^{\circ} \mathrm{K}\right)$.

Los resultados se presentan como valores positivos o negativos, que representan los flujos de los gases hacia la atmósfera, en el primer caso, o captura en el suelo, en el segundo caso.

\section{Análisis estadístico}

Para el flujo de los gases $\left(\mathrm{CH}_{4}\right.$ y $\left.\mathrm{N}_{2} \mathrm{O}\right)$ se realizaron análisis de varianza (ANOVA) y modelos lineales generales mixtos (GLM) para cumplir los requisitos de normalidad y homogeneidad de los datos. La normalidad de los 
datos se probó utilizando gráficos gg, histogramas y gráficos de cajas de residuos. La homogeneidad de los datos se probó con los gráficos de residuos versus datos esperados. Se seleccionó el modelo con los menores valores en AIC y BIC en su comparación y la máxima relación de probabilidad de registro. El carbono acumulado en el suelo (COS), se analizó mediante ANOVA de efectos principales y efectos combinados con base en el PROC GLM. El LSD de Fischer se usó para probar las medias utilizadas con un $\alpha=0,05$. Las variables ambientales y edáficas se sometieron a análisis de correlación y componentes principales, lo que determinó la asociación de variables relacionadas con los flujos de los gases $\left(\mathrm{CH}_{4}\right.$ y $\left.\mathrm{N}_{2} \mathrm{O}\right)$. Todos los análisis estadísticos se realizaron en el software estadístico Infostat 2018 (Di-Rienzo et al., 2018) y R versión 3.4.4 (Pinheiro et al., 2018).

\section{Resultados}

\section{Propiedades fisicoquímicas de suelo}

Los resultados obtenidos con relación a las propiedades físicas, no mostraron diferencias significativas $(\mathrm{p}>0,05)$ entre tratamientos. Las principales diferencias se presentaron para la densidad aparente (Da) y la humedad (Hum), con relación a la profundidad del suelo (Cuadro 2). En el primer caso, se observó que la Da mostró un

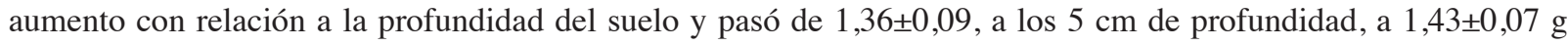
$\mathrm{cm}^{-3}$, a los $25 \mathrm{~cm}$ de profundidad. El porcentaje de Hum presentó comportamiento inverso a la Da y mostró una

Cuadro 2. Comportamiento de los parámetros físicos de suelo dentro los cuatro sistemas silvopastoriles y una pradera con solo gramínea, en el valle medio del rio Sinú, municipio de Cereté, departamento de Córdoba, Colombia. 2013.

Table 2. Behavior of the physical parameters of the soil within the four silvopastoral systems and a meadow with only grass, at the middle valley of the Sinu river, Cerete municipality, Cordoba department, Colombia. 2013.

\begin{tabular}{lcccc}
\hline Tratamientos & Da $\left(\mathbf{g ~ c m}^{-3}\right)$ & Hum $(\boldsymbol{\%})$ & Pt $(\%)$ & WFPS $(\boldsymbol{\%})$ \\
\hline Pr & $1,38 \pm 0,11$ & $18,71 \pm 1,25$ & $43,41 \pm 4,45$ & $59,85 \pm 8,65$ \\
SSP1 & $1,44 \pm 0,06^{\mathrm{a}}$ & $17,44 \pm 0,76$ & $40,89 \pm 2,40$ & $61,97 \pm 22,65$ \\
SSP2 & $1,4 \pm 0,07$ & $17,91 \pm 0,45$ & $42,48 \pm 3,13$ & $59,04 \pm 11,14$ \\
SSP3 & $1,4 \pm 0,08$ & $20,05 \pm 0,52$ & $42,76 \pm 2,64$ & $65,48 \pm 6,43$ \\
SSP4 & $1,39 \pm 0,10$ & $19,44 \pm 1,14$ & $43,12 \pm 3,50$ & $63,97 \pm 11,57$ \\
Profundidad (cm) & & & & \\
$0-5$ & $1,36 \pm 0,09^{\mathrm{a}}$ & $19,7 \pm 3,23^{\mathrm{a}}$ & $42,85 \pm 3,95$ & $63,08 \pm 13,54$ \\
$5-15$ & $1,41 \pm 0,07^{\mathrm{b}}$ & $18,61 \pm 3,65^{\mathrm{ab}}$ & $41,78 \pm 3,00$ & $63,21 \pm 13,75$ \\
$15-25$ & $1,43 \pm 0,07^{\mathrm{b}}$ & $17,8 \pm 3,83^{\mathrm{b}}$ & $42,98 \pm 2,97$ & $59,39 \pm 12,43$ \\
Sig. & $* *$ & $*$ & $\mathrm{Ns}$ & $\mathrm{Ns}$ \\
$\mathrm{R}^{2}$ & 0,46 & 0,38 & 0,38 & 0,11 \\
CV & 5,35 & 17,99 & 7,22 & 23,6 \\
Tratamiento (Tto.) & $\mathrm{Ns}$ & $\mathrm{ns}$ & $\mathrm{Ns}$ & $\mathrm{Ns}$ \\
Tratamiento (Prof.) & $*$ & $*$ & $\mathrm{Ns}$ & $\mathrm{Ns}$ \\
\hline
\end{tabular}

Da: densidad aparente $\left(\mathrm{g} \mathrm{cm}^{-3}\right)$; Hum: humedad gravimétrica $\left(\mathrm{g} \mathrm{g}^{-1}\right)$; Pt: porosidad total (\%); WFPS: porcentaje de la porosidad ocupada por agua (\%); Letras diferentes en la misma columna representan diferencias significativas; *p $<0,05$ (significativo); $* * \mathrm{p}<0,0001$ (altamente significativo); $\mathrm{R}^{2}$ : coeficiente de determinación; CV: coeficiente de variación / Da: apparent density $\left(\mathrm{g} \mathrm{cm}^{-3}\right)$; Hum: gravimetric humidity $\left(\mathrm{g} \mathrm{g}^{-1}\right)$; Pt: total porosity (\%); WFPS: percentage of porosity occupied by water (\%); Different letters in the same column represent significant differences; * $\mathrm{p}<0.05$ (significant); ** $\mathrm{p}<0.0001$ (highly significant); $\mathrm{R}^{2}$ : coefficient of determination; $\mathrm{CV}$ : coefficient of variation. 
disminución con relación a la profundidad. Por su parte, la porosidad total $(\mathrm{Pt})$ y el porcentaje de poros ocupados por agua (WFPS), presentaron un comportamiento similar a las diferentes profundidades.

Con relación a la resistencia mecánica a la penetración de las raíces en el suelo (RMP), se observó un efecto altamente significativo $(\mathrm{p}<0,01)$ para la interacción entre los tratamientos y las profundidades de muestreo, lo cual mostró que, en los primeros centímetros de profundidad, todos los tratamientos presentaron igual RMP. Sin embargo, luego de los primeros $10 \mathrm{~cm}$ de profundidad, los tratamientos asociados a los sistemas silvopastoriles (SSP) mostraron valores de RMP inferiores que el testigo modal (pradera con solo gramínea); con excepción al tratamiento SSP4 que asocia Acacia mangium, el cual mostró mayores valores de RMP a lo largo del perfil de suelo (Figura 3).

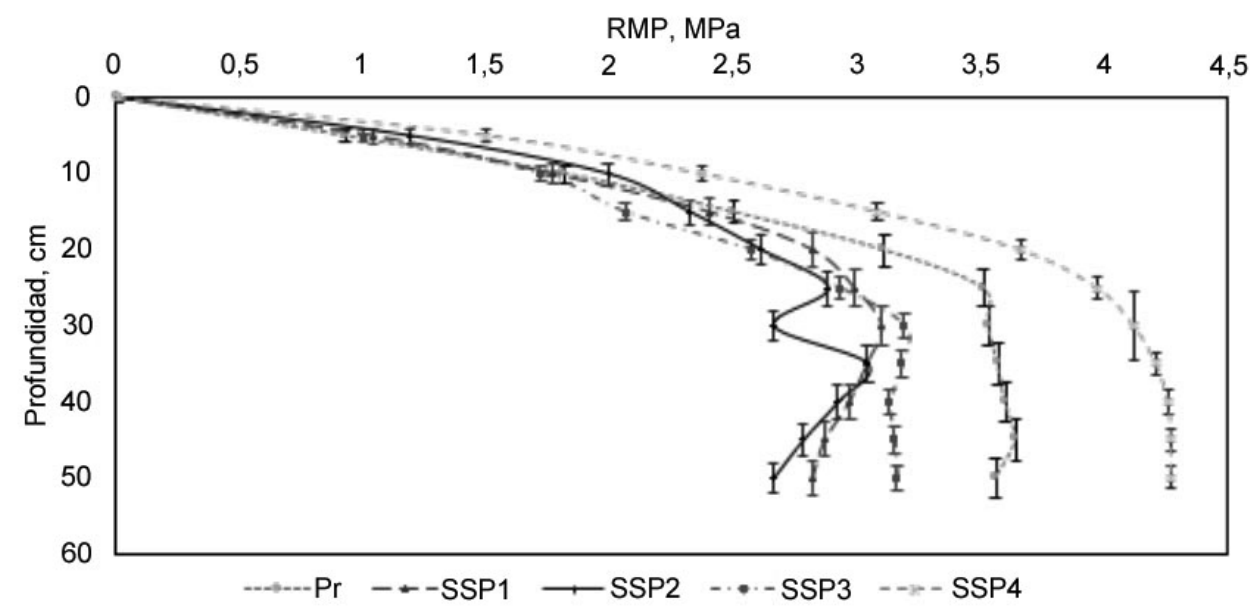

Figura 3. Resistencia mecánica a la penetración (MPa) en cuatro sistemas silvopastoriles y en una pradera de solo gramínea, en el valle medio del río Sinú, municipio de Cereté, departamento de Córdoba Colombia. 2013.

Figure 3. Mechanical resistance to penetration (MPa) in four silvopastoral systems and in a grass-only meadow, in the middle valley of the Sinú river, municipality of Cereté, department of Córdoba Colombia. 2013.

Con relación a la reacción del suelo o pH, se observó comportamiento diferencial entre los tratamientos SSP y la pradera con solo gramínea $(\mathrm{p}<0,05)$, donde el tratamiento SSP4 presentó la mayor concentración de iones $\mathrm{H}^{+}(5,83 \pm 0,22)$, siendo una condición moderadamente ácida, lo que contrasta con lo obtenido con el tratamiento SSP1 $(6,64 \pm 0,08)$. Los otros tratamientos mostraron $\mathrm{pH}$ con valores entre $6,18 \pm 0,16$ y $6,31 \pm 0,32$, considerados ligeramente ácidos (Cuadro 3).

La materia orgánica y el fósforo, no mostraron efectos de los tratamientos SSP $(p>0,05)$. Con relación a las bases intercambiables; se presentaron efectos de los tratamientos solo para el contenido de Ca intercambiable $(\mathrm{p}<0,05)$, en donde los mayores contenidos se evidenciaron en el tratamiento SSP1 $\left(10,54 \pm 0,69 \mathrm{cmol}_{(+)} \mathrm{kg}^{-1}\right)$ y los menores en el tratamiento SSP3 $\left(8,71 \pm 0,74 \mathrm{cmol}_{(+)} \mathrm{kg}^{-1}\right)$. En todos los casos la relación calcio: magnesio se presentó invertida (Cuadro 3).

\section{Carbono orgánico en el suelo (COS)}

Se encontró efecto significativo $(\mathrm{p}<0,0006)$ entre los tratamientos evaluados y el carbono orgánico acumulado en el suelo (COS). Hubo diferencia estadística significativa $(\mathrm{p}<0,0001)$ en el COS acumulado en las profundidades evaluadas. 
Cuadro 3. Contenido de nutrientes en el suelo bajo los arreglos silvopastoriles y el testigo modal (pradera con solo gramínea), en el valle medio del rio Sinú, municipio de Cereté, departamento de Córdoba, Colombia. 2013.

Table 3. Nutrient content in the soil under the silvopastoral arrangements and the modal control (grass-only meadow), at the middle valley of the Sinu river, Cerete municipality, Cordoba department, Colombia. 2013.

\begin{tabular}{|c|c|c|c|c|c|c|}
\hline Tratamientos & pH & $\begin{array}{c}\text { MO } \\
\%\end{array}$ & $\begin{array}{c}\mathbf{P} \\
\mathrm{mg} \mathrm{kg}^{-1}\end{array}$ & $\mathrm{Ca}++$ & $\begin{array}{c}\mathrm{Mg}++ \\
\operatorname{cmol}^{(+)} \mathbf{k g}^{-1}\end{array}$ & $\mathbf{K}+$ \\
\hline $\operatorname{Pr}$ & $6,18 \pm 0,16 a b$ & $4,05 \pm 0,33$ & $18,88 \pm 7,23$ & $9,85 \pm 0,42 \mathrm{ab}$ & $9,89 \pm 0,15$ & $0,84 \pm 0,09$ \\
\hline SSP1 & $6,64 \pm 0,08 \mathrm{a}$ & $4,31 \pm 0,06$ & $33,58 \pm 15,44$ & $10,54 \pm 0,69 a$ & $7,92 \pm 2,62$ & $0,9 \pm 0,26$ \\
\hline SSP2 & $6,31 \pm 0,15 \mathrm{ab}$ & $3,72 \pm 1,24$ & $26,63 \pm 7,41$ & $9,53 \pm 0,21 \mathrm{ab}$ & $6,81 \pm 5,11$ & $0,88 \pm 0,09$ \\
\hline SSP3 & $6,24 \pm 0,32 \mathrm{ab}$ & $3,84 \pm 0,12$ & $36,58 \pm 11,23$ & $8,71 \pm 0,74 b$ & $5,80 \pm 4,50$ & $1,00 \pm 0,09$ \\
\hline SSP4 & $5,83 \pm 0,22 \mathrm{~b}$ & $4,33 \pm 0,30$ & $26,61 \pm 7,72$ & $9,35 \pm 0,54 \mathrm{ab}$ & $9,15 \pm 0,22$ & $0,88 \pm 0,10$ \\
\hline $\mathrm{R}^{2}$ & 0,72 & 0,43 & 0,6 & 0,77 & 0,68 & 0,35 \\
\hline $\mathrm{CV}$ & 3,2 & 13,76 & 31,4 & 5,12 & 30,04 & 11,98 \\
\hline Sig. Trat. & $* *$ & ns & ns & $* *$ & $\mathrm{~ns}$ & Ns \\
\hline
\end{tabular}

Letras iguales no existe diferencias significativas; ${ }^{*} \mathrm{p}<0,01$ (significativo); ns: no significativo; $\mathrm{R}^{2}$ : coeficiente de determinación; $\mathrm{CV}$ : coeficiente de variación / Equal letters there are no significant differences; ${ }^{*} \mathrm{p}<0.01$ (significant); ns: not significant; $\mathrm{R}^{2}$ : coefficient of determination; $\mathrm{CV}$ : coefficient of variation.

La mayor acumulación de carbono orgánico en el suelo (COS) se presentó en los tratamientos silvopastoriles

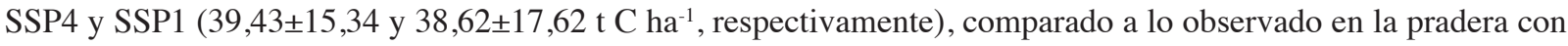
solo gramínea $\left(33,43 \pm 17,63 \mathrm{t} \mathrm{C} \mathrm{ha}^{-1}\right)$ (Figura 4a).
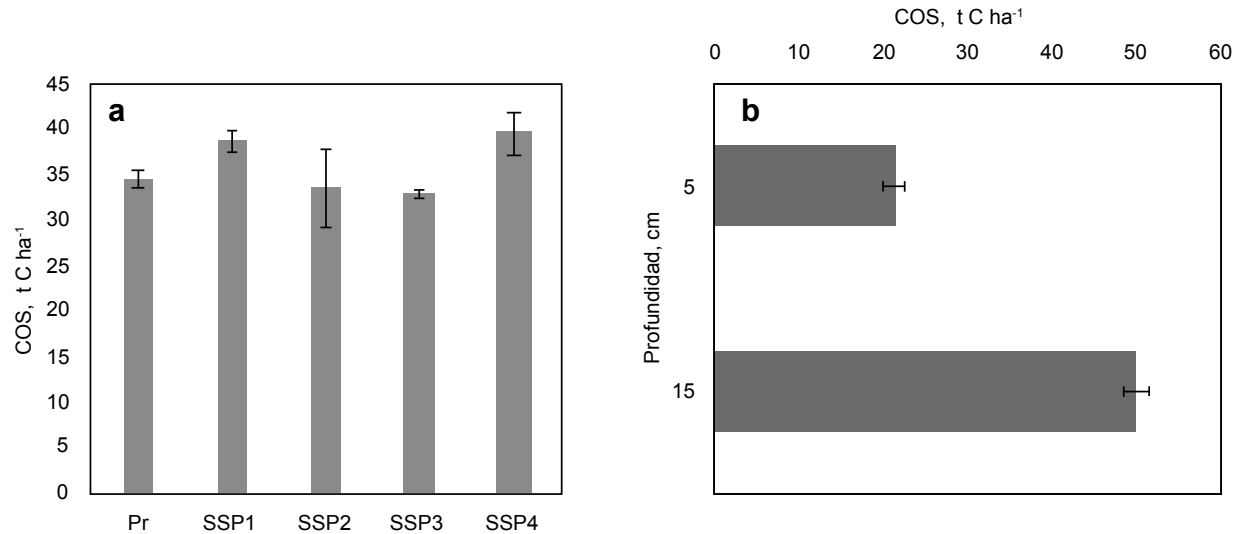

Figura 4. Carbono orgánico acumulado en le suelo en cuatro sistemas silvopastoriles y una pradera de solo gramínea, en el valle medio del río Sinú, municipio de Cereté, departamento de Córdoba Colombia, 2013.

Figure 4. Organic carbon accumulated in the soil in four silvopastoral systems and a grass-only meadow, in the middle valley of the Sinu river, Cerete municipality, Cordoba department, Colombia. 2013.

El contenido de COS aumentó en un $50 \%$ a medida que se profundizó en el perfil del suelo y varió de $21,35 \pm 3,94 \mathrm{t} \mathrm{C}^{-1}$ en los primeros $5 \mathrm{~cm}$ a $50,1 \pm 7,24 \mathrm{t} \mathrm{C}^{-1}$ en la profundidad de $15 \mathrm{~cm}$ (Figura 4b). 


\section{Metano $\left(\mathrm{CH}_{4}\right)$ y óxido nitroso $\left(\mathrm{N}_{2} \mathrm{O}\right)$}

En cuanto al flujo de metano $\left(\mathrm{FCH}_{4}\right)$ en el suelo, se observó que los distintos tratamientos silvopastoriles se comportaron como sumideros de metano durante el periodo experimental, alcanzó valores de captura de $-460 \pm 0,42$ $\mu \mathrm{g} \mathrm{CH}_{4} \mathrm{~m}^{-2} \mathrm{~h}^{-1}$, donde se destacaron los tratamientos SSP4>SSP3>SSP2. Por el contrario, los tratamientos SSP1 y Pr mostraron un comportamiento emisor con valores promedios de $540 \pm 1,56$ y $960 \pm 1,56 \mu \mathrm{g} \mathrm{CH}_{4} \mathrm{~m}^{-2} \mathrm{~h}^{-1}$ (Figura 5a).
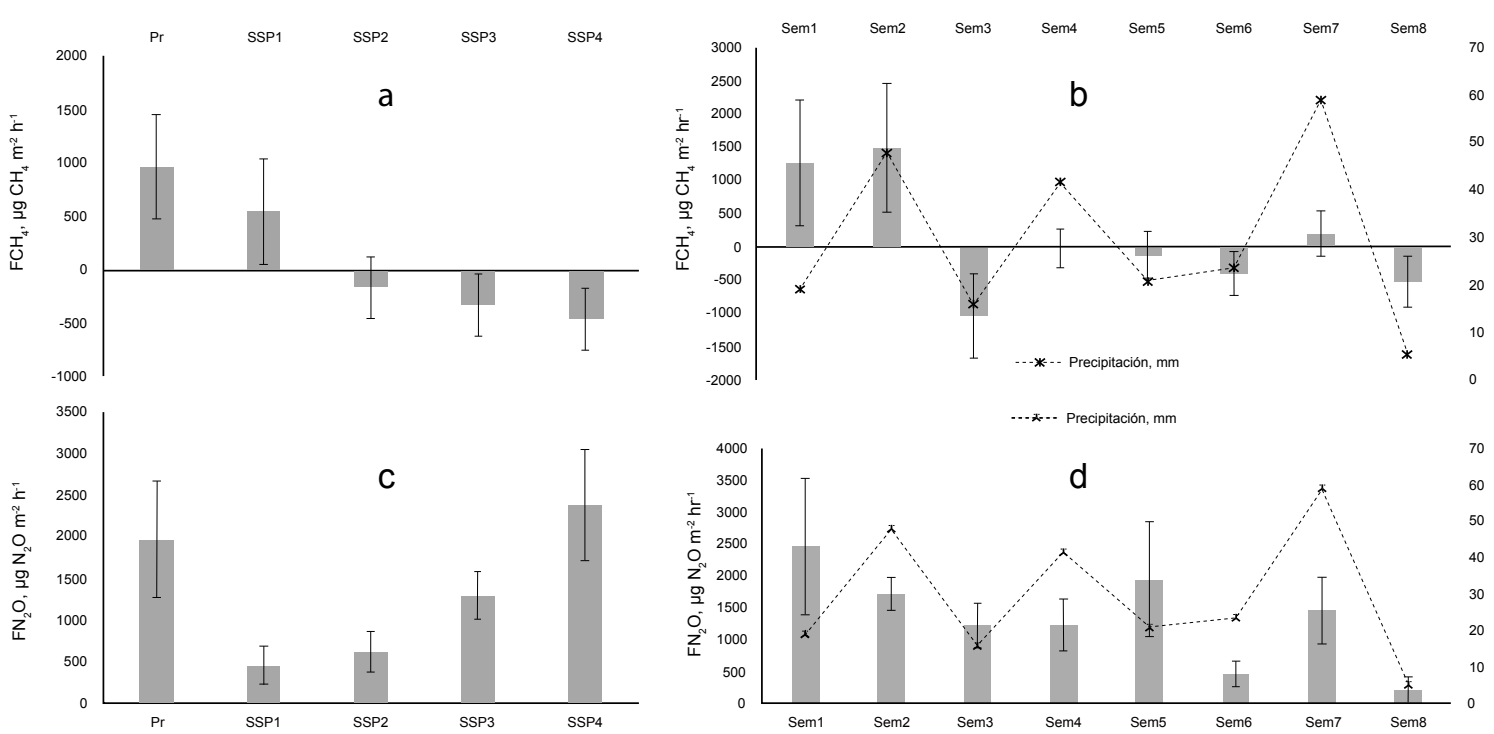

Figura 5. Flujos de gases de efecto invernadero (metano $-\mathrm{FCH}_{4}$ y óxido nitroso $-\mathrm{FN}_{2} \mathrm{O}$ ) y una pradera con solo gramínea, en el valle medio del río Sinú, municipio de Cereté, departamento de Córdoba, Colombia, bajo cuatro tratamientos silvopastoriles y su comportamiento durante ocho semanas de evaluación (septiembre a noviembre, 2013).

Figure 5. Greenhouse gas flows (methane $-\mathrm{FCH}_{4}$ and nitrous oxide $-\mathrm{FN}_{2} \mathrm{O}$ ) and a meadow with only grass, at the middle valley of the Sinu river, Cerete municipality, Cordoba department, Colombia, under four silvopastoral treatments and its behavior during eight weeks of evaluation (September to November 2013).

Los flujos de metano durante el periodo de evaluación mostraron diferencias significativas $(p<0,005)$ y en gran parte del periodo de evaluación los tratamientos fijaron metano en el suelo. A partir de la tercera semana de evaluación (primera semana octubre, 2013), los flujos de metano fueron negativos (-1030 $\left.\pm 2,09 \mu \mathrm{g} \mathrm{CH}_{4} \mathrm{~m}^{-2} \mathrm{~h}^{-1}\right)$, excepto en la séptima semana (primera semana noviembre, 2013), que presentó flujos positivos $\left(200 \pm 1,09 \mu \mathrm{g} \mathrm{CH}_{4}\right.$ $\mathrm{m}^{-2} \mathrm{~h}^{-1}$ ) (Figura 5b).

Los flujos de óxido nitroso $\left(\mathrm{N}_{2} \mathrm{O}\right)$ presentaron diferencias significativas $(\mathrm{p}<0,0001)$ entre los tratamientos (Figura 5c). La pradera de solo gramínea (Pr) y los arreglos silvopastoriles SSP4 y SSP3, presentaron las mayores emisiones de $\mathrm{N}_{2} \mathrm{O}$ hacia la atmósfera (Figura 5c). El SSP1 presentó la menor tasa de emisión de óxido nitroso $\left(460 \pm 0,60 \mu \mathrm{g} \mathrm{N}_{2} \mathrm{O} \mathrm{m}^{-2} \mathrm{~h}^{-1}\right)$. Los flujos de óxido nitroso $\left(\mathrm{N}_{2} \mathrm{O}\right)$ por semana, evidenciaron comportamiento diferencial $(\mathrm{p}<0,0001)$ entre la primera semana $\left(2470 \pm 4,58 \mu \mathrm{g} \mathrm{N}_{2} \mathrm{O} \mathrm{m}^{-2} \mathrm{~h}^{-1}\right)$ y la octava semana $\left(220 \pm 0,60 \mu \mathrm{g} \mathrm{N}_{2} \mathrm{O} \mathrm{m}^{-2} \mathrm{~h}^{-1}\right)$ (Figura 5d). 


\section{Correlaciones}

\section{Clima edáfico y ambiental}

El análisis de componentes principales y correlaciones, mostró que las variables de clima edáfico y ambiental explicaron el 58,9\% del comportamiento de los flujos de $\mathrm{GEI}\left(\mathrm{CH}_{4}\right.$ y $\left.\mathrm{N}_{2} \mathrm{O}\right)$. En la Figura 6, se presenta la participación por componente, en donde se muestra que el componente uno (1) explica en un 36,94\% el comportamiento de los flujos, mientras que el componente 2 el 21,97\%. Para el caso del componente 1, las variables de clima que mejor explican este comportamiento fueron la precipitación $(-0,59)$ y temperatura ambiental $(-0,56)$. Se evidenció que la mayoría de los flujos GEI estuvieron condicionados a estos dos parámetros ambientales. Los flujos de metano estuvieron condicionados significativamente $(\mathrm{p}<0,05)$ a la intensidad de la precipitación (Precip), la temperatura ambiental (T_Amb) y los niveles de humedad del suelo (H_Suelo) ( $\mathrm{r}=0,7 ; \mathrm{r}=0,3$ y $\mathrm{r}=0,5$, respectivamente) (Figura 6B). El análisis de correlaciones de los flujos de $\mathrm{N}_{2} \mathrm{O}$, presentó significancia $(\mathrm{p}<0,05 ; \mathrm{r}=0,2)$ respecto al contenido de humedad del suelo. Las otras variables de clima edáfico y ambiental no mostraron correlaciones significativas $(\mathrm{p}>0,05)$ respecto a los flujos de GEI.

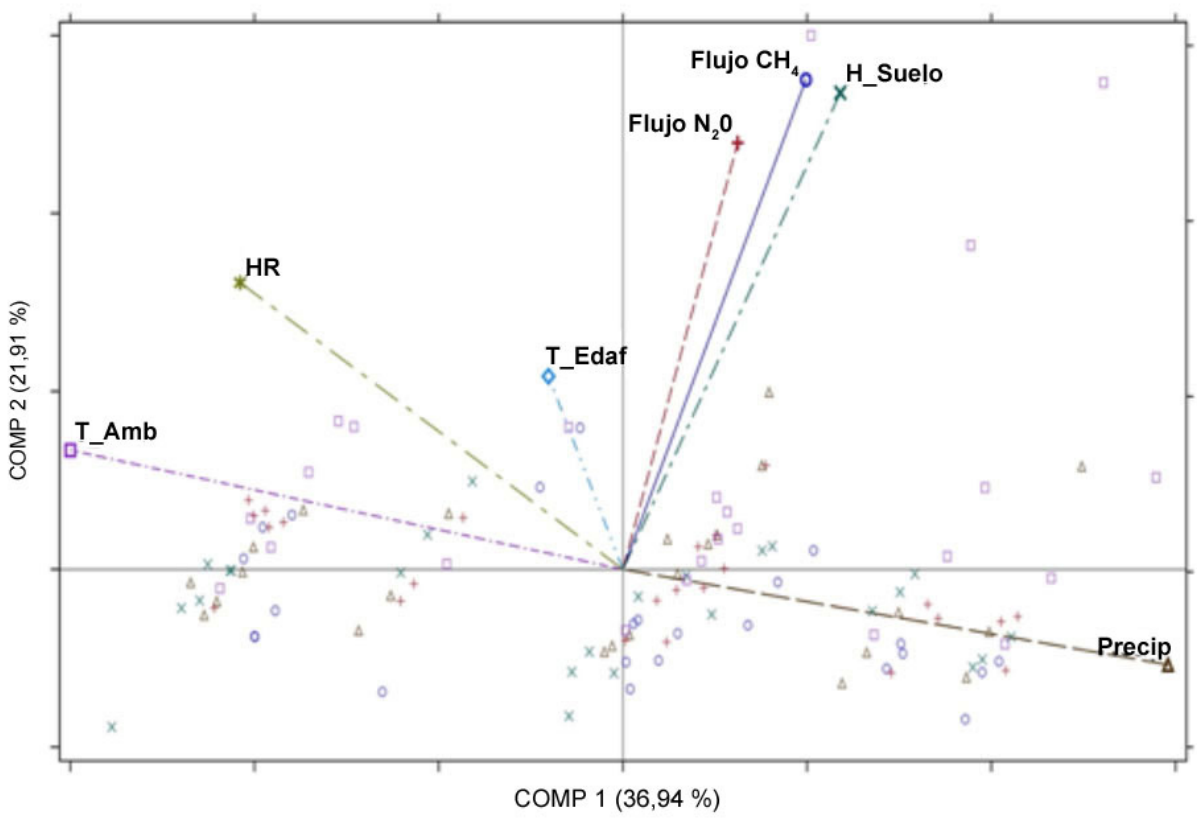

Figura 6. Análisis de componentes principales (ACP) entre los flujos de gases de efecto invernadero y de clima edáfico y ambientales bajo la influencia de cuatro sistemas silvopastoriles y una pradera con solo gramínea, en el valle medio del río Sinú, municipio de Cereté, departamento de Córdoba, Colombia. 2013.

Figure 6. Principal component analysis (PCA) between the fluxes of greenhouse gases and of edaphic and environmental climate under the influence of four silvopastoral systems and a meadow with only grass, in the middle valley of the Sinu river, Cereté municipality, Cordoba department, Colombia. 2013. 


\section{Variables fisicoquímicas de suelo}

El análisis de componentes principales para las variables fisicoquímicas del suelo (ACP), permitió evidenciar cuatro componentes principales con valores propios mayores de uno. Estos explicaron el 78,59\% de la varianza total. Para el componente uno (explicó el 30,13\% de la varianza), las propiedades con mayor peso fueron la densidad aparente $(-0,94)$, la porosidad total $(0,94)$, el calcio intercambiable $(0,78)$ y el porcentaje de la porosidad ocupada por agua $(-0,63)$. El componente dos explicó el $23,71 \%$ de la varianza, las variables con mayor peso dentro de este componente fueron el contenido de fósforo $(0,87)$, el potasio $(0,83)$ y la reacción del suelo o $\mathrm{pH}$ $(0,66)$. Para el componente tres (explicó el $15,47 \%$ de la varianza), el contenido de magnesio intercambiable $(0,86)$ y el contenido de materia orgánica $(0,68)$ tuvieron la mayor influencia; finalmente, el componente cuatro (explicó el 9,28\% de la varianza) estuvo dominado por el parámetro físico resistencia mecánica a la penetración $(0,69)$ (Figura 7).

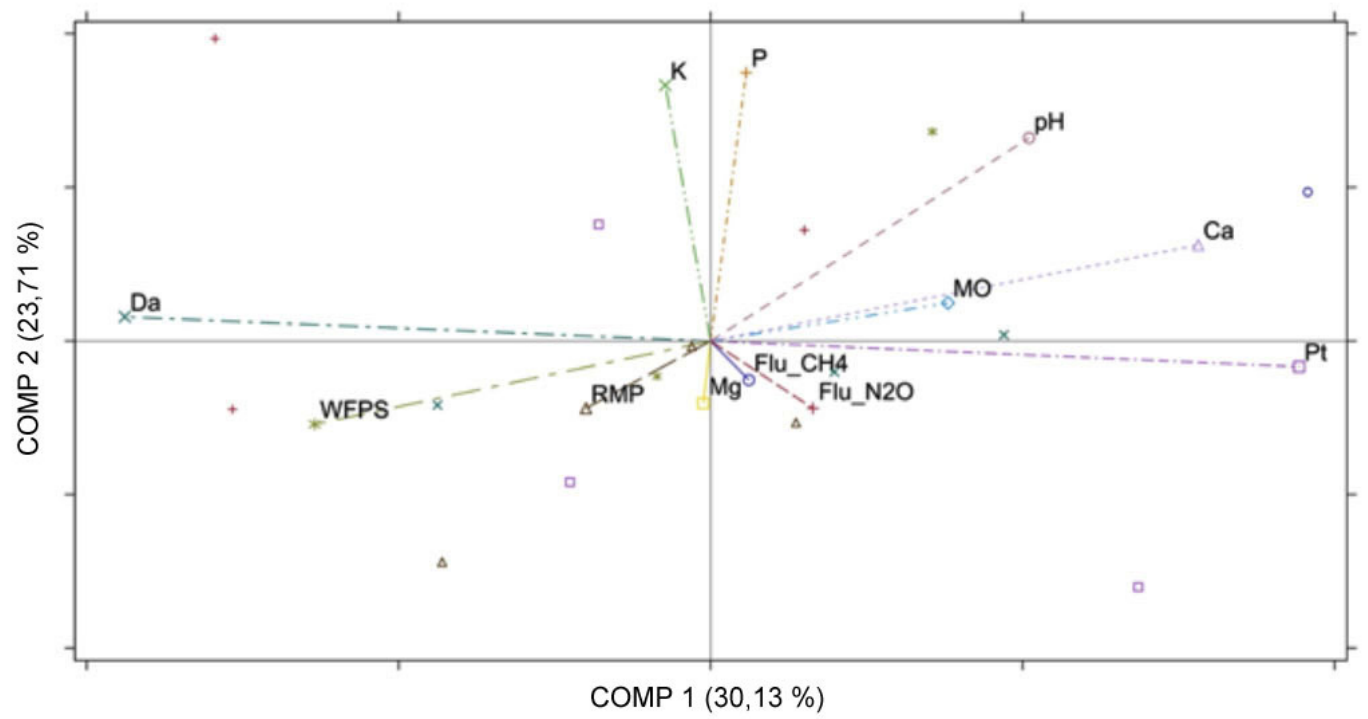

Figura 7. Análisis de componentes principales (ACP) entre los flujos de gases de efecto invernadero y las variables fisicoquímicas de suelo bajo cuatro sistemas silvopastoriles y una pradera con solo gramínea, en el valle medio del río Sinú, municipio de Cereté, departamento de Córdoba, Colombia. 2013.

Figure 7. Principal component analysis (PCA) between greenhouse gas fluxes and soil physicochemical variables under four silvopastoral systems and a grass-only meadow, at the middle valley of the Sinu river, Cerete municipality, Cordoba department, Colombia. 2013.

Se encontraron correlaciones directas significativas $(\mathrm{p}<0,05)$ entre los flujos de metano y óxido nitroso $(\mathrm{r}=0,63 ; \mathrm{r}=0,5$, respectivamente) respecto a la resistencia mecánica a la penetración. Lo anterior indica que los flujos estuvieron condicionados a los niveles de compactación del suelo debido a que el aumento de compactación generó aumento de flujos de GEI hacia la atmósfera. Respecto a las variables químicas del suelo, se evidenciaron correlaciones significativas $(\mathrm{p}<0,05)$ entre los flujos de metano, la reacción del suelo o $\mathrm{pH}(\mathrm{r}=0,56)$ y los contenidos de materia orgánica $(\mathrm{r}=0,42)$, indicaron que a $\mathrm{pH}$ altos (menor concentración de $\mathrm{H}^{+}$) y contenidos de materia orgánica altos, se disminuyeron los flujos de $\mathrm{CH}_{4}$ hacia la atmósfera. 


\section{Discusión}

El asocio de especies de gramíneas con especies arbóreas maderables (sistemas silvopastoriles), mejora las propiedades las propiedades físicas y químicas del suelo (Contreras-Santos et al., 2021). Esta afirmación se soporta en los resultados obtenidos (Cuadro 2 y 3 ) durante la evaluación del suelo. La resistencia mecánica a la penetración (RMP) se redujo dentro de los SSP luego de los primero $15 \mathrm{~cm}$ de profundidad (Figura 3) y mostró el efecto de las raíces de los árboles en el proceso de descompactación, asociado a la acumulación de materia orgánica bajo estos sistemas por las especies maderables (hojas, tallos, ramas y raíces). Los suelos bajo sistemas silvopastoriles presentan una mayor acumulación de materia orgánica en diferentes estratos del suelo, lo que favorece la conformación estructural del suelo, mayor aireación e infiltración del agua (Contreras-Santos et al., 2019; 2021; Martínez-Atencia, 2013). Los SSP se han asociado a reducción en los procesos de degradación física y química de los suelos en sistemas ganaderos (Contreras-Santos et al., 2019; Leyva et al., 2018; Vallejo et al., 2012).

Desde el punto de vista químico de suelo, se evidenció comportamiento diferencial $(\mathrm{p}<0,05)$ en el contenido de hidrogeniones o iones $\mathrm{H}^{+}$en la solución del suelo, lo cual mostró a los sistemas silvopastoriles con menor concentración que la pradera testigo (Pr), excepto en el tratamiento asociado con la especie maderable Acacia mangium (SSP4) (Cuadro 3). Resultados similares se han reportado por diferentes autores, en suelos bajo sistemas silvopastoriles que presentaron mayor acumulación de las bases intercambiable $\mathrm{Ca}^{2+}, \mathrm{Mg}^{+}$y K $\mathrm{K}^{+}$, lo que explica la reducción en la concentración de $\mathrm{H}^{+}$en suelo (p.e. Reis et al., 2009; Casals et al., 2013). La alta concentración de iones $\mathrm{H}^{+}$en el suelo dentro del SSP4, se atribuyó a la capacidad que tiene la especie leguminosa (Acacia mangium) de translocar los cationes de intercambio $\left(\mathrm{Ca}^{2+}, \mathrm{Mg}^{+}\right.$y $\left.\mathrm{K}^{+}\right)$de forma rápida a la biomasa vegetal durante su desarrollo vegetativo y a la alta concentración de aniones nitrato en el suelo a causa de sus hojas ricas en nitrógeno (Yamashita et al., 2008).

Los valores más altos dentro de los SSP de materia orgánica (MO), fósforo disponible (P) y bases de intercambio $\left(\mathrm{Ca}^{2+}, \mathrm{Mg}^{+}\right.$y K $\mathrm{K}^{+}$) (Cuadro 3), se asocian a la capacidad que tienen las especies arbóreas maderables en obtener nutrientes en estratos más profundos y colocarlos en superficie (ciclaje de nutrientes), también a los altos volúmenes de materia orgánica que son capaces de aportar estas especies (Martínez-Atencia et al., 2020). Estos resultados concuerdan con los reportados por algunos autores, quienes resaltaron que la fertilidad del suelo aumentó, pasó de 5 a $20 \%$ de acumulación de elementos mayores y bases de intercambio (P, S, Ca, Mg y K) dentro de SSP frente a pradera solo con gramínea (Casals et al., 2013; Contreras-Santos et al., 2021; Lana et al., 2016).

El carbono orgánico acumulado en el suelo (COS) presentó valores superiores al $14 \%$ dentro de los sistemas silvopastoriles (SSP) y resaltaron los valores registrados por SSP1 y SSP4 (Figura 4a), lo cual es consistente con los resultados reportados por otros estudios que encontraron mayor acumulación de MO bajo sistemas silvopastoriles (De-Stefano \& Jacobson, 2017; Dollinger \& Jose, 2019; Contreras-Santos et al., 2019; López-Santiago et al., 2018; Shi et al., 2018). Lo anterior, sustenta la afirmación de que la acumulación de carbono en el suelo está condicionado a la presencia de especies arbóreas leñosas perennes.

La mayor acumulación de COS se evidenció en la profundidad de 5 a $15 \mathrm{~cm}$, esto asociado a la capacidad de rotación de raíces finas de las especies arbóreas en los estratos más profundos, lo que facilita la acumulación de COS. Sin embargo, varios estudios han encontrado que la biomasa de raíces finas se reduce a $>30 \mathrm{~cm}$ de profundidad, asociado esto a la funcionalidad de estas, la presencia de nutrientes y el agua en estratos superficiales (ContrerasSantos et al., 2019; López-Santiago et al., 2018). Para el caso de este estudio la acumulación de COS en estratos profundos se asoció a condiciones edáficas del área de evaluación; tipo de suelo (Inceptisol, con características vérticas), con alto contenido de arcillas expansivas 2:1. Condiciones propicias para mayor acumulación de materia orgánica (MO) en estratos profundos, a causa del arrastre de material particulado o fragmentado (hojas, tallos, etc) por el viendo (caída de material por grietas, mayores a $5 \mathrm{~mm}$ ) y/o lluvias, debido a los procesos de dilatación (agrietamiento del suelo) y contracción del suelo. 
La mayor tasa de oxidación de metano (captura) en el suelo, se evidenció en el asocio de pastura con Acacia mangium (SSP4) (Figura 5a). Las mayores emisiones de metano $\left(\mathrm{CH}_{4}\right)$ estuvieron relacionadas con los periodos de lluvia (Figura 5b). Se presentaron correlaciones directas entre los flujos de metano respecto a las variables de clima edáfico - ambiental y algunas propiedades físicas del suelo, como precipitación ( $\mathrm{r}=0,7)$, humedad del suelo $(\mathrm{r}=0,5)$, temperatura ambiental $(\mathrm{r}=0,3)$, porosidad total $(\mathrm{r}=0,4)$ y resistencia mecánica a la penetración $(\mathrm{r}=0,5)$. Esto indica que altas precipitaciones aumentaron la humedad en el suelo y favorecieron las emisiones de metano hacia la atmósfera, debido a la reducción de espacios porosos, que son ocupados por el agua, lo que afectó la actividad de microorganismos metanótrofos (Ferreira, 2008; Kähkönen et al., 2002; Visscher et al., 2007). Resultados similares evidenciaron diferentes autores durante la evaluación de flujos de metano $\left(\mathrm{CH}_{4}\right)$ en el suelo bajo sistemas de producción ganaderos, quienes encontraron que una alta infiltración del agua en el suelo, baja densidad aparente, alta porosidad, alta difusión de oxígeno y baja resistencia mecánica a la penetración ( $<2 \mathrm{MPa})$ fueron condiciones que favorecieron la actividad de microorganismos metanotróficos capaces de oxidar metano en el suelo (EspinosaCarvajal et al., 2020; Luo et al., 2010; Merino et al., 2004; Rivera et al., 2018).

La tendencia de los SSP fue a reducir y/o mitigar las emisiones de $\mathrm{N}_{2} \mathrm{O}$ del suelo. Sin embargo, el asocio de una especie gramínea (M. maximus cv. Mombasa) con la especie leñosa perenne Acacia mangium (SSP4), en la evaluación de los flujos $\mathrm{N}_{2} \mathrm{O}$, mostró los valores más elevados de emisiones del suelo $\left(2380 \mu \mathrm{g} \mathrm{N}_{2} \mathrm{O} \mathrm{m}^{-2} \mathrm{~h}^{-1}\right)$ hacia la atmósfera, equivalente a cinco (5) veces más que el tratamiento SSP1 (460 $\mu \mathrm{g} \mathrm{N}_{2} \mathrm{O} \mathrm{m}^{-2} \mathrm{~h}^{-1}$ ) (Figura 5c). Estos resultados pueden atribuirse a la capacidad que tiene la especie Acacia mangium (leguminosa) de asociarse de forma simbiótica con Rhizobium y fijar nitrógeno (N) atmosférico en nódulos de raíz, lo que aumenta el ciclo de nitrógeno del suelo y muestra mayor tasa de nitrificación y alta concentración de nitratos, lo que resulta en mayores emisiones de $\mathrm{N}_{2} \mathrm{O}$ del suelo (Arai et al., 2008).

La disminución en las emisiones de $\mathrm{N}_{2} \mathrm{O}$ bajo sistemas de producción ganaderos en praderas de gramíneas con especies arbóreas leñosas perennes (SSP), está asociando a la comunidad vegetal presente y su interacción con el suelo (supresión de comunidades microbianas) (De-Carvalho et al., 2017). Los flujos de óxido nitroso $\left(\mathrm{N}_{2} \mathrm{O}\right.$ ) estuvieron condicionados a los contenidos de humedad en el suelo e indicaron que suelos con bajos contenidos de humedad se comportaron como sumideros de óxido nitroso $\left(\mathrm{N}_{2} \mathrm{O}\right)$, debido a que condiciones de suelo seco favorecieron la reducción de $\mathrm{N}_{2} \mathrm{O}$ atmosférico a $\mathrm{N}_{2}$ por las bacterias desnitrificantes (De-Carvalho et al. 2017; Dijkstra et al. 2013). Esto fue corroborado en esta investigación, ya que los flujos de $\mathrm{N}_{2} \mathrm{O}$ mostraron correlaciones significativas respecto al contenido de humedad $(\mathrm{r}=0,4)$, lo que indicó eventos de precipitación pocos o nulos generaron emisiones o disminuyeron, excepto en la semana 5 y 7 (Figura 5d).

Los resultados encontrados en esta investigación resaltaron a las variables relacionadas con procesos de compactación y movimiento de oxígeno en el suelo (Da, Pt y RMP), como las de mayor relevancia e implicación en el comportamiento de los flujos de gases de efecto invernadero del suelo evaluados. Los flujos estuvieron dominados por condiciones aeróbicas (alta disponibilidad de oxígeno en el suelo), lo que favoreció a los microrganismos metanótrofos y desnitrificantes (De-Carvalho et al. 2017; Espinosa-Carvajal et al., 2020).

\section{Conclusiones}

Los sistemas silvopastoriles (SSP) en la producción intensiva ganadera en el valle medio del rio Sinú, mejoraron significativamente las propiedades fisicoquímicas del suelo (alta porosidad, baja compactación, alta materia orgánica, alta CIC y bases de intercambio, baja concentraciones de hidrogeniones, etc.). Hubo reducción de procesos degradativos en el suelo y se generaron condiciones propicias para los microrganismos (metanotrófos y desnitrificantes) capaces de capturar metano y óxido nitroso. Sin embargo, se encontró que los flujos de gases de efecto invernadero (GEI) variaron dependiendo de las condiciones de humedad del suelo, en relación a la 
precipitación. La presencia de especies arbóreas leñosas perennes dentro de los sistemas de producción ganaderos aumentó la capacidad de almacenamiento de carbono en suelo, lo que permitió una producción sostenible.

\section{Agradecimientos}

Los autores expresan su gratitud con la Corporación Colombiana de Investigación Agropecuaria (AGROSAVIA) y el Ministerio de Agricultura y Desarrollo Rural (MADR) por financiar el proyecto "Estrategias para mejorar la competitividad y sostenibilidad de producción de carne y leche en el Caribe húmedo" bajo el convenido con MADR No. 1828 de 2014.

\section{Referencias}

Alonso, J. (2011). Los sistemas silvopastoriles y su contribución al medio ambiente. Revista Cubana de Ciencia Agrícola, 45(2), 107-115.

Arai, S., Ishizuka, S., Ohta, S., Ansori, S., Tokuchi, N., Tanaka, N, \& Hardjono, A. (2008). Potential $\mathrm{N}_{2} \mathrm{O}$ emissions from leguminous tree plantation soils in the humid tropics. Global Biogeochemical Cycles, 22(2), Article GB2028. https:// doi.org/10.1029/2007gb002965

Bray, R. H., \& Kurtz, L. T. (1945). Determination of total, organic, and available forms of phosphorus in soils. Soil Science, 59(1), 39-46. https://doi.org/10.1097/00010694-194501000-00006

Casals, P., Romero, J., Rusch, G. M., \& Ibrahim, M. (2013). Soil organic C and nutrient contents under trees with different functional characteristics in seasonally dry tropical silvopastures. Plant and Soil, 374(1-2), 643-659. https://doi. org/10.1007/s11104-013-1884-9

Charmley, E., Williams, S. R. O., Moate, P. J., Hegarty, R. S., Herd, R. M., Oddy, V. H., Reyenga, P., Staunton, K. M., Anderson, A., \& Hannah, M. C. (2016). A universal equation to predict methane production of forage-fed cattle in Australia. Animal Production Science, 56(3), Article 169. https://doi.org/10.1071/an15365

Chapman, H. D. (2016). Cation-exchange capacity In A. G. Norman (Ed.), Methods of soil analysis: Part 2 Chemical and microbiological properties (Chapter 57, pp. 891-901). American Society of Agronomy. https://doi.org/10.2134/ agronmonogr9.2.c6

Chu, H., Hosen, Y., \& Yagi, K. (2007). NO, $\mathrm{N}_{2} \mathrm{O}, \mathrm{CH}_{4}$ and $\mathrm{CO}_{2}$ fluxes in winter barley field of Japanese Andisol as affected by N fertilizer management. Soil Biology and Biochemistry, 39, 330-339. https://doi.org/10.1016/j.soilbio.2006.08.003

Contreras-Santos, J. L., Martínez-Atencia, J., Cadena-Torres, J., \& Fallas-Guzmán, C. K. (2019). Evaluación del carbono acumulado en suelo en sistemas silvopastoriles del Caribe Colombiano. Agronomía Costarricense, 44(1), 29-41. https://doi.org/10.15517/rac.v44i1.39999

Contreras-Santos, J. L., Martínez-Atencia, J., \& Falla-Guzman, C. (2021). Carbono acumulado en raíces de especies vegetales en sistemas silvopastoriles en el Norte de Colombia. Revista de Ciencias Ambientales, 55(1), 52-69. https://doi. org/10.15359/rca.55-1.3

De-Carvalho, A. M., de Oliveira, W. R. D., Ramos, M. L. G., Coser, T. R., de Oliveira, A. D., Pulrolnik, K., Souza, K. W., Vileta, L., \& Marchão, R. L. (2017). Soil $\mathrm{N}_{2} \mathrm{O}$ fluxes in integrated production systems, continuous pasture and Cerrado. Nutrient Cycling in Agroecosystems, 108(1), 69-83. https://doi.org/10.1007/s10705-017-9823-4 
De-Stefano, A., \& Jacobson, M. G. (2017). Soil carbon sequestration in agroforestry systems: a meta-analysis. Agroforestry Systems, 92, 285-299. https://doi.org/10.1007/s10457-017-0147-9

Di-Rienzo, J., F. Casanoves, M. Balzarina, L. Gonzalez, M. Tablada, \& C. Robledo. (2018). Infostat versión 2018. Universidad Nacional de Córdoba.

Dijkstra F. A., Morgan J. A., Follett R. F., \& Lecain D. R. (2013) Climate change reduces the net sink of CH4 and N2O in a semiarid grassland. Global Change Biology, 19(6), 1816-1826. https://doi.org/10.1111/gcb.12182

Dollinger, J., \& Jose, S. (2019). Agroforestry for soil health. Agroforestry Systems, 93(3), 1205-1205. https://doi.org/10.1007/ s10457-018-0223-9

Espinosa-Carvajal, M., Contreras-Santos, J., Torres, J., Martínez-Atencia, J., Jaramillo-Barrios, C., \& Hurtado, M. (2020). Flujos de metano en suelos con coberturas de pastos en el norte de Colombia. Agronomía Mesoamericana, 31(2), 291-309. https://doi.org/10.15517/am.v31i2.38387

Ferreira, O. (2008). Flujos de gases de efecto invernadero, potencial de calentamiento global y evaluación de emergía del sistema agroforestal quesungual en el sur de Lempira, Honduras [Tesis de Maestría, no publicada], Universidad Nacional de Colombia.

Food and Agriculture Organization. (2017). Soil organic carbon: the hidden potential. http://www.fao.org/3/i6937e/i6937e.pdf

Gerber, P. J., Steinfeld, H., Henderson, B., Mottet, A., Opio, C., Dijkman, J., Falcucci, A., \& Tempio, G. (2013). Tackling climate change through livestock - A global assessment of emissions and mitigation opportunities. Food and Agriculture Organization. Retrieved December 6, 2019, from http://www.fao.org/3/a-i3437e.pdf

Hernández, J. H., \& Corona, L. (2018). El metano y la ganadería bovina en México: ¿Parte de la solución y no del problema? Agroproductividad, 11(2),46-51.

Hendershot, W., Lalande, H., \& Duquette, M. (2007). Ion exchange and exchangeable cations In M. R. Carter, \& E. G. Gregorich, Soil Sampling and Methods of Analysis (2 ${ }^{\text {nd }}$ Ed. pp. 197-207). Canadian Society of Soil Science.

Holdridge, L. (2000). Ecología basada en zonas de vida. Instituto Interamericano de Cooperación para la Agricultura.

Instituto Geográfico Agustín Codazzi. (2006). Métodos analíticos del laboratorio de suelos (6 ${ }^{\mathrm{a}}$ Ed.). Imprenta Nacional de Colombia.

Jose, S., \& Dollinger, J. (2019). Silvopasture: a sustainable livestock production system. Agroforestry Systems, 93(1), 1-9. https://doi.org/10.1007/s10457-019-00366-8

Kähkönen, M. A., Wittmann, C., Ilvesniemi, H., Westman, C. J., \& Salkinoja-Salonen, M. (2002). Mineralization of detritus and oxidation of methane in acid boreal coniferous forest soils: seasonal and vertical distribution and effects of clear-cut. Soil Biology and Biochemistry, 34(8), 1191-1200. https://doi.org/10.1016/s0038-0717(02)00056-1

Lana, Â. M. Q., Lana, R. M. Q., Lemes, E. M., Reis, G. L., \& Moreira, G. H. F. A. (2016). Influence of native or exotic trees on soil fertility in decades of silvopastoral system at the Brazilian savannah biome. Agroforestry Systems, 92, $415-424$. https://doi.org/10.1007/s10457-016-9998-8

Leyva, S., Baldoquin, A., \& Reyes, M. (2018). Propiedades de los suelos en diferentes usos agropecuarios, Las Tunas, Cuba. Revista Ciencias Agrícolas, 35(1), 36-47. http://dx.doi.org/10.22267/rcia.183501.81.

López-Santiago, J. G., Casanova-Lugo, F., Villanueva-López, G., Díaz-Echeverría, V. F., Solorio-Sánchez, F. J., MartínezZurimendi, P., Aryal, D. R., \& Chay-Canul, A. J. (2018). Carbon storage in a silvopastoral system compared to that 
in a deciduous dry forest in Michoacán, Mexico. Agroforestry Systems, 93(1), 199-211. https://doi.org/10.1007/ s10457-018-0259-x

Luo, J., de Klein C. A. M., Ledgard, S. F., \& Saggar, S. (2010) Management options to reduce nitrous oxide emissions from intensively grazed pastures: a review. Agriculture, Ecosystems \& Environment, 136(3-4), 282-291. https://doi. org/10.1016/j.agee.2009.12.003

Martínez-Atencia, J. (2013). Producción y descomposición de hojarasca en sistemas silvopastoriles de estratos múltiples y su efecto sobre propiedades bioorgánicas del suelo en el valle medio del Río Sinú [Tesis de Doctorado, Universidad Nacional de Colombia], Repositorio de la Universidad Nacional de Colombia. https://repositorio.unal.edu.co/handle/ unal/20938

Martínez-Atencia, J., Loaiza-Usuga, J. C., Osorio-Vega, N. W., Correa-Londoño, G., \& Casamitjana-Causa, M. (2020). Leaf litter decomposition in diverse silvopastoral systems in a neotropical environment. Journal of Sustainable Forestry, 39(7), 710-729. https://doi.org/10.1080/10549811.2020.1723112

Merino, A., Pérez-Batallón, P., \& Macías, F. (2004). Influencia del uso y manejo agrícola sobre la dinámica de CH4 del suelo en el norte de España. Edafología, 11(2), 207-219.

Montagnini, F., Somarriba, E., Murgueitio, E., Fassola, H., \& Eibl, B. (2015). Sistemas Agroforestales. Funciones Productivas, Socioeconómicas y Ambientales (Serie Técnica, Informe Técnico 402). Centro Agronómico Tropical de Investigación y Enseñanza. https://espace.library.uq.edu.au/data/UQ_296869f/UQ296869f_OA.pdf?Expires=1616010826\&KeyPair-Id=APKAJKNBJ4MJBJNC6NLQ\&Signature=U7U5GYxfoF9CFtx $\sim \mathrm{mXnDr8E6yrphnMQR4blCA47KJG6Fmt-}$ wDGejrLgzDFIVzhR7uiu75hL4vPSQCM5WWA0uRGRZTzpGv3zzVKvNxhAPgWvXSLB6gcURKC60A4E187dM2TwTNb5zkZxn8axCRPq8FNtF2Hb 99Z1mHB1CTj8LmE3c5rwkM8SHTSr9pwd3t1jLQ Yt7LmFLGK8sAKEq 2Inu4RKUuH0 3FdUUNM-Vo8Heyfr4g-9jcBFlGbpkUlfrkC6WJGAqAff-0qhR7uhVdkpoevMPfC7nfL4H6FtlJhP8 VbMyZee2gxVr6KW5 e3VzwL1k4BPw3xW9ArXpRVDmufQ_\#page=276

Murgueitio-Restrepo, E., Barahona-Rosales, R., Flores-Estrada, M. X., Chará-Orozco, J. D., \& Rivera-Herrera, J. E. (2016). Es posible enfrentar el cambio climático y producir más leche y carne con sistemas silvopastoriles intensivos. Ceiba, 54(1), 23-30. https://doi.org/10.5377/ceiba.v54i1.2774

Nelson, D., \& Sommers, L. (1983) Total carbon, organic carbon and organic matter. In A. Page, R. Miller, \& D. Kenney (Eds.), Methods of soil analysis. Part 2 Chemical and microbiological properties (2 ${ }^{\text {nd }}$ Ed. pp. 539-579-430). American Society of Agronomy and Soil Science Society of America. https://doi.org/10.2134/agronmonogr9.2.2ed.c29

Organización de las Naciones Unidas para la Alimentación y la Agricultura, \& Grupo Técnico intergubernamental de Suelos. (2015). Estado Mundial del Recurso Suelo (EMRS) - Resumen Técnico. Recuperado el 9 de septiembre de 2018, de http://www.fao.org/3/a-i5126s.pdf

Pachauri, R. K., Meyer, L. A., \& Stocker, T. (Eds.) (2014). IPCC 2014: Cambio climático 2014: Informe de síntesis. Contribución de los Grupos de Trabajo I. II y III al Quinto Informe de evaluación del panel intergubernamental sobre el cambio climático. Intergovernmental Panel on Climate Change. https:/www.ipcc.ch/site/assets/uploads/2018/02/ SYR_AR5_FINAL_full_es.pdf

Pastrana, V. I., Reza, G. S., Espinosa, C. M., Suárez, P. E., \& Díaz, A. E. (2011). Efecto de la fertilización nitrogenada en la dinámica del óxido nitroso y metano en Brachiaria humidicola (Rendle) Schweickerdt. Ciencia y Tecnología Agropecuaria, 12(2), 134.https://doi.org/10.21930/rcta.vol12_num2_art:223 
Pérez-Ramírez, S., Ramírez, M. I., Jaramillo-López, P. F., \& Bautista, F. (2013). Contenido de carbono orgánico en el suelo bajo diferentes condiciones forestales: reserva de la biosfera mariposa monarca, México. Revista Chapingo Serie Ciencias Forestales y del Ambiente, 19(1), 157-173. https://doi.org/10.5154/r.rchscfa.2012.06.042

Pinheiro, J., Bates, D., DebRoy, S., \& Sarkar, D. (2018). Nlme: linear and nonlinear mixed effects models. R package version 3.1-137. R Core Team. Retrieved June 21,9019, from https://CRAN.R-project.org/package=nlme

Reis, G. L., Lana, Â. M. Q., Maurício, R. M., Lana, R. M. Q., Machado, R. M., Borges, I., \& Neto, T. Q. (2009). Influence of trees on soil nutrient pools in a silvopastoral system in the Brazilian Savannah. Plant and Soil, 329(1-2), 185-193. https://doi.org/10.1007/s11104-009-0144-5

Rivera, J. E., Chará, J., \& Barahona, R. (2018). $\mathrm{CH}_{4}, \mathrm{CO}_{2}$ and $\mathrm{N}_{2} \mathrm{O}$ emissions from grasslands and bovine excreta in two intensive tropical dairy production systems. Agroforestry Systems, 93(3), 915-928. https://doi.org/10.1007/s10457-018-0187-9

Rondón M. (2000). Land use and balances of greenhouse gases in Colombian tropical savannas [Doctor Thesis no published]. Cornell University.

Shi, L., Feng, W., Xu, J., \& Kuzyakov, Y. (2018). Agroforestry systems: Meta-analysis of soil carbon stocks, sequestration processes, and future potentials. Land Degradation \& Development, 29(11), 3886-3897. https://doi.org/10.1002/ $\operatorname{ldr} .3136$

Soil Survey Staff. (2014). Keys to soil taxonomy (12 ${ }^{\text {th }}$ Ed.). USDA-Natural Resources Conservation Service. https://www.nrcs. usda.gov/Internet/FSE_DOCUMENTS/nrcs142p2_051546.pdf

Vallejo, V. E., Roldán, F., \& Dick, R. P. (2012). Soil enzymatic activities and microbial biomass in an integrated agroforestry chronosequence compared to monoculture and a native forest in Colombia. Biology and Fertility of Soils, 46(6), 577587. https://doi.org/10.1007/s00374-010-0466-8

Visscher, A., Boeckx, P., \& Van-Cleemput, O. (2007). Artificial methane sinks In Reay, D. S., Hewitt, C. N., Smith, K. A., \& Grace J. (Eds.), Greenhouse gas sinks (pp. 184-200). CABI Agriculture and Bioscience. https://doi. org/10.1079/9781845931896.0184

Yamashita, N., Ohta, S., \& Hardjono, A. (2008). Soil changes induced by Acacia mangium plantation establishment: Comparison with secondary forest and Imperata cylindrica grassland soils in South Sumatra, Indonesia. Forest Ecology and Management, 254(2), 362-370. https://doi.org/10.1016/j.foreco.2007.08.012 